\title{
Donner du sens à la citoyenneté plutôt que l'enseigner
}

\section{Andrée Tabouret-Keller}

\section{(2) OpenEdition \\ 1 Journals}

\section{Édition électronique}

URL : http://journals.openedition.org/trema/1728

DOI : 10.4000/trema. 1728

ISSN : 2107-0997

\section{Éditeur}

Faculté d'Éducation de l'université de Montpellier

\section{Édition imprimée}

Date de publication : 1 octobre 1999

Pagination : 81-84

ISSN : 1167-315X

\section{Référence électronique}

Andrée Tabouret-Keller, « Donner du sens à la citoyenneté plutôt que l'enseigner », Tréma [En ligne],

15-16 | 1999, mis en ligne le 01 octobre 1999, consulté le 02 mai 2019. URL : http:// journals.openedition.org/trema/1728; DOI : 10.4000/trema.1728

Ce document a été généré automatiquement le 2 mai 2019.

Trema 


\title{
Donner du sens à la citoyenneté plutôt que l'enseigner
}

\author{
Andrée Tabouret-Keller
}

1 La rédaction du texte qui suit se situe dans l'après-coup du colloque et de la Table Ronde à laquelle j'ai participé. L'argument que j'avais proposé avant la tenue du colloque était le suivant :

- La citoyenneté n'est pas une affaire de compétence (chacun naît déjà citoyen) mais de paroles à dire pour que les inscriptions fondatrices ne restent pas lettre morte.

- Le sens que pourra prendre la citoyenneté pour les jeunes dépend du courage qu'auront les adultes, qui sont des modèles pour eux (parents, maîtres, etc.), à assumer leurs propres conduites en matière de citoyenneté, soit qu'ils en évitent les devoirs, soit qu'ils y attachent de l'importance. De ce point de vue, les exercices de citoyenneté que l'on a pu proposer (exercices de civilité et de civisme dans les écoles) sont au mieux des entreprise naïves, voire niaises, au pire des entreprises perverses si elles restent des exercices que l'adulte propose aux jeunes - parmi d'autres - sans exercer de regard critique sur ses propres engagements de citoyen mais aussi à l'égard des démarches citoyennes contemporaines (parmi bien d'autres : abstention des électeurs, récurrence des grèves... il y en a eu pas mal en début d'année scolaire 1998 dans les lycées !).

- Le respect de l'âge des enfants et de leurs propres manières d'appréhender le monde et la société doit être au centre des démarches d'une éducation citoyenne. La conception en terme d'apprentissage peut faire perdre de vue que la connaissance enseignée risque de rester un discours parmi les innombrables discours que notre école s'échine à faire apprendre, bons à obtenir une bonne note, à passer un examen. Il n'y a donc pas de " vocabulaire ", ou même de " compétence langagière » en général, mais uniquement des paroles assumées et adressées.

2 Nous ne formerons pas des citoyens à contre-courant de la société si partout ailleurs les valeurs citoyennes sont dénigrées, voire déniées.

3 Les propos que j'ai, en sus, pu tenir au cours de la Table Ronde, et que je complète dans la forme écrite de ma communication en m'appuyant sur les réflexions de Paul Ricœur (en 
particulier le chapitre "Politique et totalitarisme" de son ouvrage La critique et la conviction (1995, pp. 147-75), visent à mettre en lumière un certain nombre de difficultés apparues dans nos journées.

La citoyenneté dont nous parlons ici, se développe dans un type d'Etat qui s'appuie sur un gouvernement démocratique. Dans le cas de la France, comme dans d'autres pays voisins, des éléctions mettent en place des représentants du peuple, parlant et décidant au nom de groupes plus ou moins importants. "Comment », interroge Ricœur, "éduquer à l'adhésion critique des citoyens qui sont dans la situation de ne jamais pouvoir engendrer à partir d'euxmêmes le politique?»(p.157)

Une telle question a été éludée jusqu'ici ; sans pouvoir la développer, elle mérite au moins d'être posée. Elle est doublée d'une autre question plus particulièrement liée à la formation d'enfants et de jeunes gens à l'exercice de la citoyenneté : c'est celle de la complexité des niveaux de gouvernance. En démocratie, les mécanismes de pouvoir sont complexes.

FUMAT Y., GENZLING A. \& PERFETTINI M. : Éducation civique - $3^{\mathrm{e}}$ (Nouveau programme de 1985). Paris, F. Nathan, 1989, 127 p., p. 11

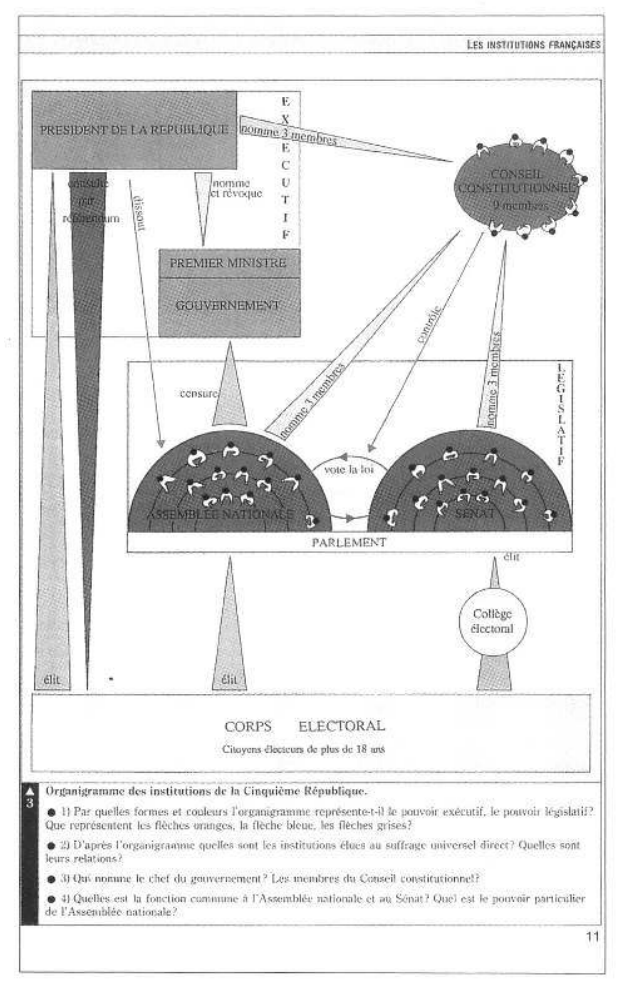

6 Dans une classe de 35 élèves, dans une école de 500 élèves, voire dans un lycée de 2000 élèves, la complexité des phénomènes de participation à la prise de décision couvre déjà un large éventail de processus, mais elle peut encore être analysée. A l'échelle de la souveraineté d'Etat, les facteurs de la prise de décisions deviennent indéchiffrables à cause de la complexification des sphères d'appartenance qui gouvernent la société civile. Les gouvernements régionaux, locaux, et le gouvernement supra-national européen, qui se met tant bien que mal en place, correspondent à des niveaux différents de compétence, à des hiérarchies d'autorité aussi (autorité : une autre notion que nous n'avons pas examinée). «Nous ne fonctionnons pas à tout moment comme citoyen; nous nous comportons de 
temps en temps seulement comme citoyen - par exemple, lorsque nous allons voter - mais beaucoup plus souvent comme producteur ou comme conservateur» (p. 158). C'est ce qui explique, précise Ricœur, que l'on puisse avoir l'impression que le politique n'est qu'une de nos activités parmi d'autres. Mais c'est là perdre de vue, selon moi, que lors même que nous ne nous occupons pas de politique, l'Etat demeure l'englobant de toutes les sphères d'appartenance à l'égard desquelles nous faisons allégeance. "Nous n'avons pas à l'égard de l'Etat une allégeance comme nous en avons une à l'égard de l'université ou d'un club de football; le lien de citoyenneté est toujours présupposé par tous les autres... » (p. 158).

Il me semble que dans les discours tenus lors de ce colloque, l'on procédait comme si la continuité entre les exercices de citoyenneté à l'école et les pratiques institutionnelles de la citoyenneté dans le cadre de l'Etat était évidente : une telle continuité n'est cependant ni évidente, ni même assurée. "La ‘cité` au sens politique n'est pas réductible à la somme des 〈cités〉 auxquelles nous appartenons, à la somme des «sphères〉 d'allégeance constitutives de la société civile. » (p. 159).

Une autre confusion me semble avoir pesé sur nos discussions, entre appartenance à une institution et appartenance à un corps politique par la citoyenneté. En Allemagne, jusqu'à fort récemment, même si vous étiez résident de longue durée, vous ne deveniez jamais citoyen allemand, mais, dans certaines circonstances, un résident étranger pouvait participer à une élection. En France, ce n'est pas le cas. «L'espace de juridiction d'un Etat, est ce qui montre que l'appartenance au corps politique est véritablement première» (p. 160); cette appartenance est manifestée par la citoyenneté.

Dans le second point de mon argument, j'insistais sur la fonction de modèle remplie par les adultes, indispensable à tenir si l'on veut que l'enfant, ou le jeune, puisse repérer ce qu'on lui demande et quelles conséquences dans la vie quotidienne cela entraîne. Sans développer ce point, je remarque, jusque dans le déroulement de nos travaux, qu'une conduite aussi simple, en matière d'égalité citoyenne, que le respect des prises de paroles et du temps imparti à chacun, est simplement ignorée. Les enfants ont bon dos: non seulement, on s'abstient de leur donner un exemple qui puisse être argumenté, voire suivi, mais on se donne bonne conscience en énumérant tout ce qu'il faut leur apprendre. A être respectueux, patients, utiles, coopérants, responsables, si bien que l'on vient à se demander si ce zèle manifesté à si haute et insistante voix par les adultes, d'enseigner exhaustivement des compétences citoyennes, ne porte pas en lui la dispense pour l'adulte, d'en faire de son côté, ne fut-ce qu'un peu plus que de dresser des programmes pour les écoles.

Et comme il est question de compétences et d'apprentissages, ma brève intervention illustre quelques possibilités autour des notions suivantes. La représentation est à la fois une notion et un exercice difficiles : il est possible de l'illustrer dans la pratique en faisant l'expérience de la pérennité de la fonction, assortie du caractère passager de celui qui la remplit (une fonction de représentation particulière - délégué de classe, par exemple remplie par un autre, d'année en année). La citoyenneté institutionnelle, illustrée par les droits différents dont relèvent tels ou tels groupes d'immigrés ou, aujourd'hui de réfugiés, en France où ils ont l'autorisation de rester trois mois, ou dans leur pays d'origine. Les groupes d'appartenance : ils correspondent aux exemples le plus souvent donnés ici car ils permettent d'illustrer assez facilement les devoirs, parfois contradictoires, qui peuvent résulter des allégeances qu'ils exigent (le port du foulard est un exemple particulièrement complexe d'allégeances contradictoires). L'expérience la 
plus difficile, tant pour les enfants que pour les adultes, reste certes de penser la complexité et l'intrication des niveaux de décisions.

\section{BIBLIOGRAPHIE}

RICCEUR P. : La critique et la conviction. Entretien avec François Azouvi et Marc de Launay. Paris, Calmann-Lévy, 1995.

\section{RÉSUMÉS}

L'auteur soutient la position selon laquelle la citoyenneté n'est pas une affaire de compétence chacun naît déjà citoyen - mais de paroles à dire pour que les inscriptions fondatrices ne restent pas lettre morte.

The author asserts the position that citizenship is not a matter of skills, (the position arguing that we are all citizens by birth), but that instead it is a matter of expressing in speech and actions the ideals of fundamental texts so that they be not left to gathering dust.

INDEX

Mots-clés : citoyenneté institutionnelle, groupe d'appartenance, représentation Keywords : institutional citizenship, Membership group

\section{AUTEUR}

\section{ANDRÉE TABOURET-KELLER}

Université Louis Pasteur de Strasbourg 